\title{
Examining the effects of gaming and guessing on script concordance test scores
}

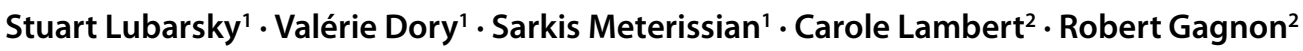

Published online: 12 June 2018

(c) The Author(s) 2018

\begin{abstract}
Introduction In a script concordance test (SCT), examinees are asked to judge the effect of a new piece of clinical information on a proposed hypothesis. Answers are collected using a Likert-type scale (ranging from -2 to +2 , with ' 0 ' indicating no effect), and compared with those of a reference panel of 'experts'. It has been argued, however, that SCT may be susceptible to the influences of gaming and guesswork. This study aims to address some of the mounting concern over the response process validity of SCT scores.

Method Using published datasets from three independent SCTs, we investigated examinee response patterns, and computed the score a hypothetical examinee would obtain on each of the tests if he 1) guessed random answers and 2) deliberately answered ' 0 ' on all test items.

Results A simulated random guessing strategy led to scores 2 SDs below mean scores of actual respondents (Z-scores -3.6 to -2.1 ). A simulated 'all-0' strategy led to scores at least 1 SD above those obtained by random guessing (Z-scores -2.2 to -0.7 ). In one dataset, stepwise exclusion of items with modal panel response ' 0 ' to fewer than $10 \%$ of the total number of test items yielded hypothetical scores 2 SDs below mean scores of actual respondents.

Discussion Random guessing was not an advantageous response strategy. An 'all-0' response strategy, however, demonstrated evidence of artificial score inflation. Our findings pose a significant threat to the SCT's validity argument. 'Testwiseness' is a potential hazard to all testing formats, and appropriate countermeasures must be established. We propose an approach that might be used to mitigate a potentially real and troubling phenomenon in script concordance testing. The impact of this approach on the content validity of SCTs merits further discussion.
\end{abstract}

Keywords Assessment $\cdot$ Clinical reasoning $\cdot$ Script concordance

\section{What this paper adds}

Random guessing is not an advantageous response strategy in script concordance testing. Deliberate selection of the ' 0 ' response on all test items demonstrates evidence of artificial score inflation, posing a significant threat to the SCT's validity argument. Limiting the proportion of SCT items with modal panel response ' 0 ' can mitigate the effects of an 'all-

Editor's Note: Commentary by: E. Custers, https://doi.org/10. 1007/s40037-018-0437-6

Stuart Lubarsky

stuart.lubarsky@mcgill.ca

1 Centre for Medical Education, McGill University, Montreal, Canada

2 Centre de pédagogie appliquée aux sciences de la santé (CPASS), Université de Montréal, Montreal, Canada
0' response strategy, but could also have adverse effects on content validity.

\section{Introduction}

The Script Concordance Test (SCT) is used to assess a specific aspect of clinical reasoning competence: clinical data interpretation (CDI) under conditions of uncertainty [1]. Its theoretical foundation emerged from the cognitive psychology literature out of a larger debate about the nature of expertise [2]. According to current theories of expertise development, individuals judged to be 'experts' in a given field possess neither superior generic problem-solving skills, nor an enhanced capacity for memory retrieval. The real hallmark of an expert is the structured manner in which his or her knowledge is organized in the mind [3]. Expert knowledge in a given domain is interlinked, or 'chunked', in such 
Table 1 Example of a Script Concordance Test item featuring 3 questions nested within one case. Clinical scenario: You are evaluating a 63year-old woman with left-sided weakness in the Emergency Department of your hospital

\begin{tabular}{llllll}
\hline If you were thinking ...: & And then you find ...: & \multicolumn{3}{l}{ Your hypothesis becomes ...: } \\
\hline Q1. Brain abscess & Patient had dental work 1 month ago & -2 & -1 & 0 & +1 \\
Q2. Stroke & Patient uses a vaginal oestrogen cream once daily & -2 & -1 & 0 & +1 \\
Q3. Brain metastasis & Normal non-contrast CT head & -2 & -1 & 0 & +1 \\
\hline
\end{tabular}

-2 : Ruled out or almost ruled out; -1 : Less likely; 0: Neither more nor less likely; +1 : More likely; +2 : Certain or almost certain

a way as to facilitate its rapid access, as an integrated unit, at the right time and in the proper context.

In medicine, some of these knowledge 'chunks' are referred to as 'illness scripts' [4]. Illness scripts are bounded networks of medical knowledge that activate automatically in a physician's mind early on during clinical encounters, guiding subsequent reasoning through a given case [5]. Illness scripts allow physicians to integrate new incoming information with existing knowledge, recognize patterns and irregularities in symptom complexes, identify similarities and differences between disease states, and make predictions about how diseases are likely to unfold [6].

The SCT aims to examine respondents' illness scripts under a microscopic lens [1, 7]. In an SCT, examinees are asked to render a judgment-generally using a 5-point Likert-type scale-about the direction (positive or negative) and magnitude (strong or weak) of the association between a new piece of clinical information and a given hypothesis (Tab. 1). A ' 0 ' response option is available for respondents to indicate their belief that the new bit of clinical data has no effect on the hypothesis provided. To generate scores, examinees' responses are compared with those of members of a pre-selected expert panel. ${ }^{1}$ Script concordance hinges on an inference that examinees with 'higher-quality' illness scripts interpret data and make judgments in uncertain situations that increasingly concord with those of experienced clinicians given the same clinical scenarios [10].

There are, however, few empirical data to support the claim that SCT offers useful insights into the way that medical knowledge is organized into scripts in the minds of examinees. Lubarsky et al. [11] conducted a review of the literature evaluating the construct validity of the script concordance method, following an established approach for analyzing validity data from five categories: content, response process, internal structure, relations to other variables, and

\footnotetext{
${ }^{1}$ For each SCT item, a maximum score of 1 is given for the response chosen by most of the experts (i.e., the modal response). Other responses are given partial credit, depending on the fraction of experts choosing them. Responses not selected by experts receive zero. An examinee's total score for the test is the sum of the credit obtained for each of the questions, divided by the total obtainable credit for the test, and multiplied by 100 to derive a percentage score. Psychometricians support the use of this type of system, referred to as 'aggregate scoring' $[8,9]$.
}

consequences [12]. While the authors found moderate to strong validity evidence in several categories, they concluded that the validity of SCT scores is only weakly supported by evidence pertaining to response process, which entails the unearthing of data elucidating the relationship between an assessment's intended construct and the thought processes and response actions of its examinees [11].

In one computer-based study using the script concordance format, subjects were asked to gauge the effect (i.e. more likely, less likely, or no effect) of new pieces of information on a series of diagnostic hypotheses [13]. Subjects responded significantly faster when they were presented clinical information that was either highly typical or irreconcilable with the given hypothesis than when they were presented information that was merely atypical, suggesting that processing times were influenced by the 'degree of compatibility' between new clinical information and relevant activated scripts-in other words, by the strength of association between acquired health-related concepts in the subjects' minds. SCT investigators have also pointed to the observation that SCT scores consistently tend to increase with increasing level of training to indirectly support their claim that SCT provides valid information about illness script development [7].

Since the publication of the literature review by Lubarsky et al. [11], other investigators have furnished evidence that, in fact, undermines the response process validity of SCT score interpretations [14-17]. For example, as part of a larger examination of the current SCT scoring system, Lineberry and colleagues [16] investigated the effect on SCT scoring of deliberately responding ' 0 ' to all items on the test. Based on a re-analysis of data from previouslypublished work by Bland, Kreiter, and Gordon [18], the investigators demonstrated that a hypothetical examinee who simply endorsed the scale midpoint for every item would outperform most other examinees using the scale as it was intended.

The point Lineberry and his colleagues [16] were trying to make in devising this inauthentic scenario was that the SCT is susceptible to construct-irrelevant response tendencies, however unlikely. As they point out, the possibility that the SCT could be 'gamed' in such a manner poses a potentially serious threat to the response process validity of SCT score interpretation, since test-wise examinees are 
apt to catch on that defaulting to the ' 0 ' response would lead to artificial score inflation. However, the results of the Lineberry study, which involved re-analysis of a single set of scores obtained from an SCT using a suboptimal 8-member panel, need to be interpreted with caution. Prior work on SCT has shown that, in high-stakes settings, at least 15 panel members are required to obtain stable estimates of the reliability of scores, and that reliability becomes severely compromised when panels consist of fewer than 10 members [19].

The present study aims to shed further light on the particular response tendencies exhibited by SCT examinees, and to address some of the mounting concern over the response process validity of SCT scores. Using published datasets from three independent SCTs, each with a score key devised by reference panels comprising 15 members or more, we sought to examine the 'epidemiology' of selected response options by SCT examinees, and to verify the legitimacy of the claim that SCT scores are susceptible to the influences of gaming and guesswork. Our specific research questions were:

1. How frequently are individual Likert-type scale responses $(-2,-1,0,+1$, or +2$)$ selected by actual SCT examinees?

2. How would a hypothetical examinee selecting random answers to every item on a specific SCT perform compared with an examinee completing the same test as intended?

3. How would a hypothetical examinee deliberately responding ' 0 ' to every item on a specific SCT perform compared with an examinee completing the same test as intended?

4. If influences of guessing (as per Research Question \#2) or gaming (as per Research Question \#3) are detected on a specific SCT, can item manipulation mitigate these influences?

\section{Methods}

\section{Databases}

To conduct our analyses, we used data culled from three independent, previously-published SCT studies [20-22]. In each study, a panel of at least 15 expert members was used to develop the scoring key for the test. Cronbach's alpha coefficients were considered to be adequate in all three studies. Test characteristics for each of the SCTs analyzed in this study are shown in Tab. 2.
Table 2 Test characteristics

\begin{tabular}{lccc}
\hline & $\begin{array}{l}\text { Radiation- } \\
\text { oncology [20] }\end{array}$ & $\begin{array}{l}\text { Neurology } \\
{[21]}\end{array}$ & $\begin{array}{l}\text { General } \\
\text { surgery [22] }\end{array}$ \\
\hline Participants & & & \\
$N$ clerks & 70 & 8 & 0 \\
$N$ residents & 38 & 41 & 202 \\
(R1-R5) & & & \\
$N$ panellists & 42 & 16 & 21 \\
Items & & & \\
$N$ cases & 30 & 24 & 43 \\
$N$ questions & 70 & 79 & 131 \\
Proportion of items with panel modal response: & \\
-2 & $6 \%$ & $9 \%$ & $26 \%$ \\
-1 & $38 \%$ & $27 \%$ & $40 \%$ \\
0 & $24 \%$ & $29 \%$ & $27 \%$ \\
+1 & $22 \%$ & $29 \%$ & $3 \%$ \\
+2 & $10 \%$ & $6 \%$ & $3 \%$ \\
Cronbach's & 0.90 & 0.79 & 0.85 \\
alpha & & &
\end{tabular}

\section{Analyses}

To examine the frequency of selection of individual Likerttype scale responses by actual examinees, we calculated the percentage of each answer selected by participants on the three SCTs under investigation.

To examine the influence on scoring of completing an SCT using a guessing strategy, we derived 100 random answer combinations for each of the three SCT datasets using the Excel random function and computed descriptive statistics. For each study, we conducted the analysis once using a traditional 5-point scale, and a second time using a proposed alternative 3-point scale [18].

To examine the influence on scoring of completing an SCT using an 'all 0' strategy, we calculated the score a hypothetical examinee would obtain on each of the three SCTs if he or she were to consistently answer ' 0 ' on all test items.

To examine the feasibility of mitigating the effects of gaming on score inflation, we devised a strategy using data from Lambert et al.'s [20] database, which resulted in the highest mean score for a hypothetical examinee adopting the 'all 0' response tactic. Since questions for which the modal panel response is 0 award full credit to examinees who also respond 0 , we surmised that reducing their number would be the most efficient way to limit the benefits associated with an 'all 0 ' gaming strategy. We therefore excluded these questions one by one, recalculating the hypothetical examinee's final score after each item was discarded. For each iteration of the test, we also calculated z-scores, scores and associated descriptive statistics for actual respondents, and Cronbach's alpha as an indicator of test reliability.

Given the hypothetical nature of the experiment and the lack of potential to cause harm to human individuals, formal 
Table 3 Distribution of responses according to sub-groups of respondents

\begin{tabular}{llrllll}
\hline & & \multicolumn{1}{c}{-2} & \multicolumn{1}{c}{-1} & 0 & +1 & +2 \\
\hline Radiation- & Clerks & $10 \%$ & $28 \%$ & $28 \%$ & $22 \%$ & $12 \%$ \\
oncology & Residents & $9 \%$ & $27 \%$ & $22 \%$ & $28 \%$ & $14 \%$ \\
& Panellists & $11 \%$ & $27 \%$ & $29 \%$ & $20 \%$ & $13 \%$ \\
\multirow{2}{*}{ Neurology } & Clerks & $13 \%$ & $30 \%$ & $23 \%$ & $25 \%$ & $10 \%$ \\
& Residents & $10 \%$ & $30 \%$ & $23 \%$ & $25 \%$ & $12 \%$ \\
& Panellists & $10 \%$ & $28 \%$ & $31 \%$ & $23 \%$ & $8 \%$ \\
\multirow{3}{*}{ General } & Residents & $19 \%$ & $38 \%$ & $23 \%$ & $14 \%$ & $6 \%$ \\
\hline
\end{tabular}

review by our institution's ethics review committee was waived.

\section{Results}

\section{Frequency of actual responses (Tab. 3)}

In the three SCTs under analysis, the 0-response represented $21-31 \%$ of test answers, with no differences observed according to expertise level (Tab. 3). Response patterns were similar for two of the tests, i.e. the extreme points of the Likert-type scale $(-2$ and +2$)$ were selected less frequently; most answers were equally distributed between the $-1,0$ and +1 points. In the general surgery test, answers were spread mostly across the $-2,-1$ and 0 points.

\section{Guessing (Tab. 4)}

A simulated guessing strategy based on use of a 5-point Likert-type scale (denoted 'Random 5' in Tab. 4) led to mean scores ranging between 2.42 and 3.60 standard deviations below the mean scores of actual examinees. A simulated guessing strategy based on use of a 3-point Likerttype scale (denoted 'Random 3' in Tab. 4) led to scores ranging between 1.63 and 2.00 standard deviations below the mean scores of actual examinees.

\section{Gaming (Tab. 4)}

A simulated gaming strategy, whereby a hypothetical examinee was assumed to deliberately answer 0 on every question, resulted in scores ranging between 0.73 and 2.2 standard deviations below the mean scores of actual examinees.

\section{Mitigating effects of excluding questions with a modal panel response of 0 (Tab. 5; Fig. 1)}

Excluding questions with a modal panel response of 0 had a linear effect on the $\mathrm{z}$ score of a hypothetical examinee using an all 0 strategy, with minimal impact on the internal consistency of the test. Based on calculations from
Table 4 Scores of actual respondents, a hypothetical examinee providing random answers (i.e. guessing), and a hypothetical examinee using an 'all 0' strategy (i.e. gaming)

\begin{tabular}{|c|c|c|c|c|c|}
\hline & Groups & $N$ & Mean & SD & $\begin{array}{l}\mathrm{Z} \\
\text { score* }\end{array}$ \\
\hline \multicolumn{6}{|c|}{ Radiation-oncology } \\
\hline \multirow{3}{*}{$\begin{array}{l}\text { Actual } \\
\text { respondents }\end{array}$} & Clerks & 70 & 51.6 & 8.2 & \\
\hline & Residents & 38 & 71.2 & 9.5 & \\
\hline & $\begin{array}{l}\text { All exami- } \\
\text { nees }\end{array}$ & 108 & 59.3 & 9.7 & \\
\hline \multirow[t]{2}{*}{ Guessing } & Random 5 & 100 & 35.8 & 4.0 & -2.42 \\
\hline & Random 3 & 100 & 43.7 & 2.7 & -1.63 \\
\hline Gaming & All 0 & & 52.2 & & -0.73 \\
\hline \multicolumn{6}{|l|}{ Neurology } \\
\hline \multirow{3}{*}{$\begin{array}{l}\text { Actual } \\
\text { respondents }\end{array}$} & Clerks & 8 & 63.6 & 6.3 & \\
\hline & Residents & 41 & 70.3 & 8.8 & \\
\hline & $\begin{array}{l}\text { All exami- } \\
\text { nees }\end{array}$ & 49 & 67.9 & 8.8 & \\
\hline \multirow[t]{2}{*}{ Guessing } & Random 5 & 100 & 35.9 & 4.4 & -3.6 \\
\hline & Random 3 & 100 & 50.9 & 4.1 & -2.0 \\
\hline Gaming & All 0 & & 53.2 & & -1.7 \\
\hline \multicolumn{6}{|c|}{ General surgery } \\
\hline $\begin{array}{l}\text { Actual re- } \\
\text { spondents }\end{array}$ & Residents & 202 & 68.1 & 7.4 & \\
\hline \multirow[t]{2}{*}{ Guessing } & Random 5 & 100 & 42.6 & 3.0 & -3.4 \\
\hline & Random 3 & 100 & 53.8 & 3.2 & -1.9 \\
\hline Gaming & All 0 & & 51.7 & & -2.2 \\
\hline
\end{tabular}

*Z scores indicate by how many standard deviations a score deviates from the mean

the Lambert et al. [20] dataset, limiting the proportion of questions eliciting a panel modal response of ' 0 ' to fewer than $7 \%$ ensured that the all-0 strategy resulted in scores below 2 standard deviations from the mean.

\section{Discussion}

In this study, we sought to examine the general response patterns of script concordance test (SCT) examinees, and to explore the potential effects on SCT scores of 1) guessing (i.e. selecting responses in a random fashion) and 2) gaming (i.e. selecting responses in a deliberate manner to manipulate the scoring system). Calculating the scores a hypothetical examinee would obtain if he had engaged in either of these tactics, and comparing these with the scores obtained by actual examinees on several published SCTs, we found several interesting results.

First, we found that in all three SCTs under investigation examinees tended to avoid selecting responses at the extremes of the Likert-type scale (i.e., +2 and -2 ), a response tendency that has been suspected but never empirically verified. This response pattern was similar for examinees at all levels of expertise (i.e. from clerkship student 
Table 5 Effect on actual respondent scores (using data from Lambert et al. [22]) of excluding questions with a modal panel response of 0 oneby-one, and recalculating the examinee's final score after each item was discarded. In shaded rows, a pass-fail threshold of 2 standard deviations below the mean would ensure that gamers fail the test

\begin{tabular}{|c|c|c|c|c|c|c|c|}
\hline $\begin{array}{l}N \text { of questions with } \\
\text { a modal panel response } \\
\text { of } 0\end{array}$ & $\begin{array}{l}\% \text { Questions with a modal } \\
\text { panel response of } 0\end{array}$ & $\begin{array}{l}\text { 'All-0' score for } \\
\text { a hypothetical } \\
\text { examinee }\end{array}$ & $\begin{array}{l}\text { Mean score of } \\
\text { actual exami- } \\
\text { nees }\end{array}$ & Min & Max & $\begin{array}{l}\text { All } 0 \\
\text { z score }\end{array}$ & $\begin{array}{l}\text { Cronbach's } \\
\text { alpha }\end{array}$ \\
\hline 22 & $24.4 \%$ & 52.2 & 62.0 & 37.3 & 84.1 & -0.90 & 0.904 \\
\hline 21 & $23.3 \%$ & 51.0 & 61.2 & 37.3 & 83.0 & -0.95 & 0.902 \\
\hline 20 & $22.2 \%$ & 49.9 & 60.5 & 36.2 & 81.8 & -1.00 & 0.900 \\
\hline 19 & $21.1 \%$ & 48.8 & 59.7 & 35.8 & 80.7 & -1.04 & 0.896 \\
\hline 18 & $20.0 \%$ & 47.7 & 59.2 & 35.8 & 79.6 & -1.12 & 0.893 \\
\hline 17 & $18.9 \%$ & 46.6 & 58.5 & 35.8 & 78.5 & -1.18 & 0.892 \\
\hline 16 & $17.8 \%$ & 45.5 & 57.9 & 35.8 & 77.4 & -1.27 & 0.891 \\
\hline 15 & $16.7 \%$ & 44.4 & 57.4 & 35.6 & 76.3 & -1.34 & 0.891 \\
\hline 14 & $15.6 \%$ & 43.3 & 56.8 & 35.5 & 75.2 & -1.42 & 0.888 \\
\hline 13 & $14.4 \%$ & 42.2 & 56.3 & 35.4 & 74.1 & -1.52 & 0.884 \\
\hline 12 & $13.3 \%$ & 41.0 & 55.8 & 34.3 & 73.6 & -1.61 & 0.882 \\
\hline 11 & $12.2 \%$ & 39.9 & 55.1 & 34.1 & 72.7 & -1.67 & 0.881 \\
\hline 10 & $11.1 \%$ & 38.8 & 54.2 & 33.7 & 71.6 & -1.72 & 0.879 \\
\hline 9 & $10.0 \%$ & 37.7 & 53.6 & 33.9 & 70.8 & -1.87 & 0.876 \\
\hline 8 & $8.9 \%$ & 36.6 & 52.9 & 32.6 & 70.4 & -1.94 & 0.875 \\
\hline 7 & $7.8 \%$ & 35.5 & 52.3 & 31.5 & 69.3 & -1.99 & 0.871 \\
\hline 6 & $6.7 \%$ & 34.4 & 51.7 & 31.1 & 68.2 & -2.08 & 0.868 \\
\hline 5 & $5.6 \%$ & 33.3 & 51.4 & 30.4 & 68.2 & -2.19 & 0.865 \\
\hline 4 & $4.4 \%$ & 32.3 & 50.6 & 30.0 & 67.1 & -2.22 & 0.865 \\
\hline 3 & $3.3 \%$ & 31.0 & 50.1 & 30.0 & 66.8 & -2.37 & 0.860 \\
\hline 2 & $2.2 \%$ & 29.9 & 49.3 & 29.4 & 65.6 & -2.43 & 0.858 \\
\hline 1 & $1.1 \%$ & 28.8 & 48.6 & 29.2 & 65.1 & -2.52 & 0.857 \\
\hline 0 & $0.0 \%$ & 27.7 & 47.7 & 28.3 & 64.0 & -2.53 & 0.859 \\
\hline
\end{tabular}

to practising physician). In general surgery (but not neurology or radiation oncology), both residents and practising surgeons were more likely to select responses on the negative than the positive spectrum of the Likert scale, hinting that response tendencies might vary according to discipline of practice (although test-specific effects, rather than discipline-specific effects, certainly cannot be excluded in this small study). In none of the studies was the 0-response more frequently selected than +1 or -1 responses; we found no evidence of an all-0 response strategy, or even a predilection toward 0-responses, in any of the SCTs we examined.

Second, we found that a simulated random guessing strategy using a 5-point Likert-type scale led to scores at least 2 standard deviations (SDs) below mean scores of actual respondents on three separate SCT datasets. If the common cut-off point for normative standard setting, i.e. $-2 \mathrm{SD}$ from the mean, were to be used as a pass/fail marker, a guessing strategy on a 5-point Likert-type scale would not prove to be advantageous.

A simulated random guessing strategy using a 3-point aggregate Likert-type scale, on the other hand, led to higher scores than those calculated using the 5-point Likert-type scale. This finding offers a potentially useful contribution to the ongoing discussion over the optimal method for scoring an SCT [18, 23]. Bland and colleagues [18], for example, found few differences in psychometric performance between five different SCT scoring methods (including use of an aggregate 5-point and aggregate 3-point Likert-type scale). However, if indeed use of the two scales leads to comparable reliability estimates, our finding would suggest that the 5-point Likert-type scale should be maintained in light of its greater capacity to withstand the influences of random guessing.

Third, we found that a simulated 'all 0' strategy, whereby the ' 0 ' response was assumed to have been purposely selected as an answer to every item on the test, led to scores at least $1 \mathrm{SD}$ above those obtained by random guessing. Although we found no evidence of use of an 'all 0' strategy in actual respondents at any level of training, this tactic would appear to be effective: in 2 out of 3 studies it artificially inflated scores beyond the -2 SD point recommended for normative standard-setting, and in one study it resulted in scores similar to those obtained by clerks who completed the exam faithfully. This finding replicates the results of the recent hypothetical experiment conducted by Lineberry and 


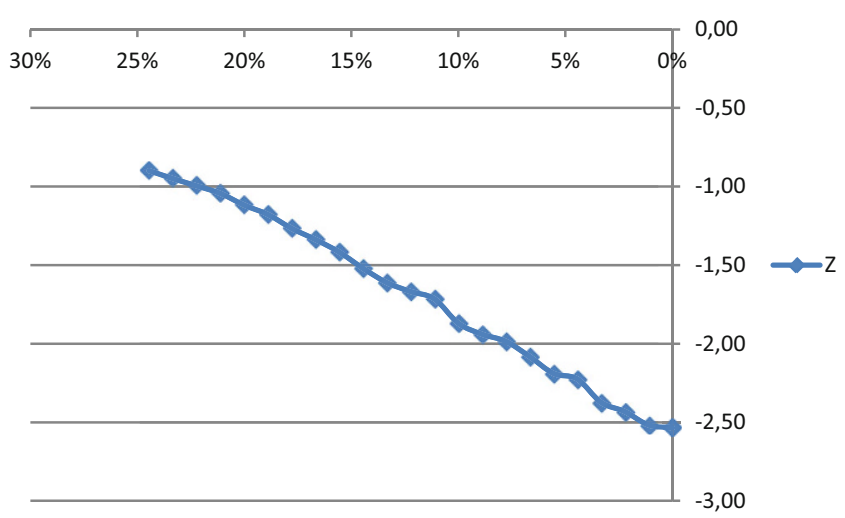

Fig. 1 Z-score of a 'gamer' on subtests with different proportions of questions with modal panel responses of 0

colleagues [16], and indeed poses a significant threat to the SCT's validity argument.

'Testwiseness' is a potential hazard to all testing formats $[24,25]$. Appropriate countermeasures for any test, including the script concordance test, must therefore be established. In this study, we found that limiting the proportion of items with a modal panel response of ' 0 ' to fewer than $10 \%$ of the total number of test items mitigated the effects of the 'all 0' strategy in one SCT. Our finding that gaming of the SCT can be thwarted by diligent test construction complements the recent findings of See, Tan and Lim [26], who tackled the problem from a slightly different perspective. Examining the effect of deliberate avoidance of +2 and -2 responses (rather than deliberate selection of the 0 response) on test results, See and colleagues [26] found that increasing the proportion of questions with extreme modal answers to $50 \%$ overcame score inflation due to abstention from selecting extreme responses.

The impact on the test's content validity of restricting items with modal 0-responses to fewer than $10 \%$ of the total test items merits further discussion. Current guidelines for SCT construction already recommend that test developers generate one-and-a-half times the amount of questions they actually intend to use, because a certain proportion of items with poor item-total correlations are expected to be eliminated post hoc from the final version of the exam $[7,27]$. Removal of an additional proportion of items with modal 0-responses would entail further reduction in sampling from the test's content blueprint, potentially compromising the test's content validity. Deliberate attempts during the test construction phase to limit the number of items expected to yield modal 0-responses from the panel imposes an added constraint on test development.

Moreover, forfeiting 0-response items would concede one of SCT's unique testing properties: assessment of an examinee's ability to discern relevant clinical data from those that have little to no bearing on a given hypothesis, a skill necessary for coping with the ambiguities of daily practice.
Trade-off on content validity resulting from discriminate inclusion and exclusion of test items from a question bank, however, is by no means a challenge unique to script concordance testing, and can be minimized through judicious selection of items and careful consideration of a test's purpose and target group [28].

\section{Limitations}

To examine the effects of guessing on SCT scores, we devised the rather unrealistic scenario in which a hypothetical examinee guesses the answers to all of the questions on the test in random fashion. Although actual examinees are unlikely to respond to test items in such a completely haphazard manner, the demonstration of benefits in scoring through the use of such an extreme strategy clearly would have undermined the validity of SCT score interpretations. Indeed, the use of a deliberate all-0 response tactic-another extreme, improbable test-taking strategy, originally conceived by Lineberry and colleagues [16] — was found to be advantageous.

This study has several other limitations. Although for the purpose of this study we examined several datasets of scores obtained from SCTs created according to published guidelines, all analyses were conducted retrospectively. Furthermore, we did not study SCTs developed for use in disciplines other than medicine, such as pharmacy [29], veterinary medicine [30], and nursing [31]. Finally, the strategy we developed for offsetting the effects of a gaming strategy was used on a single dataset only, and therefore our conclusion that mode- 0 responses should represent fewer than $10 \%$ of an SCT's test items cannot necessarily be extrapolated to any other version of the test. However, we believe that the approach we adopted (i.e., stepwise exclusion of 0 -modal items until a-2 SD threshold is attained) has merit, and might be implemented in other cases of SCT to mitigate what we have found to be a real and troubling phenomenon in script concordance testing. The approach could be used to examine the effects of eliminating questions with other modal answers (e.g. questions that elicit $+1,+2,-1$, and -2 modal responses), as well.

\section{Future avenues of research}

Ultimately, the response process validity of SCT scores requires further exploration. An investigation of examinees' inclination to avoid extreme responses warrants particular attention: Is this tendency ethnically or culturally mediated, as Lineberry and colleagues [16] have postulated? Is it a discipline-specific predisposition? Or is there simply no meaningful difference in the minds of examinees between 
the 'extreme' (+2 and -2$)$ and 'less extreme' (+1 and -1$)$ responses?

Perhaps the tendency to avoid the ends of the scale could be tempered by careful wording of the instructions presented to examinees prior to SCT administration (i.e. simply by encouraging consideration of full use of the scale throughout completion of an SCT). Or perhaps the problem resides in SCT item development, i.e. in the challenge posed by creating items that reliably elicit responses ranging across the spectrum of the Likert-type scale. SCT response tendencies could be investigated in greater detail by asking examinees to submit written justifications for their responses, by asking them to think out loud as they respond to SCT items, or by interviewing them just after completion of an SCT. Phrasing of SCT instructions, as well as differences in wording of the scale anchors, likely have a significant influence on examinee response patterns, but thus far have been only minimally examined and warrant further attention [32].

Finally, in order to further examine the effects of guessing and various gaming strategies (all-0, extreme-avoidance, etc.) on SCT scores, a more authentic simulation of a 'partial' rather than a 'complete' guessing strategy could be modelled, and prospective studies could be undertaken using tests deliberately developed to contain restricted numbers of items with modal response of 0 and/or higher numbers of items with modal responses at the extremes of the Likert scale. Studies investigating the relationship between SCT scores and other measures of knowledge organization could also shed light on whether the proposed inference between the two is substantiated, providing further evidence for or against the response process validity of script concordance testing.

Open Access This article is distributed under the terms of the Creative Commons Attribution 4.0 International License (http:// creativecommons.org/licenses/by/4.0/), which permits unrestricted use, distribution, and reproduction in any medium, provided you give appropriate credit to the original author(s) and the source, provide a link to the Creative Commons license, and indicate if changes were made.

\section{References}

1. Charlin B, van der Vleuten C. Standardized assessment of reasoning in contexts of uncertainty: the script concordance approach. Eval Health Prof. 2014;27:304-19.

2. Schmidt HG, Norman GR, Boshuizen HPA. A cognitive perspective on medical expertise: theory and implications. Acad Med. 1990;65:611-21.

3. Lemieux M, Bordage G. Propositional versus structural semantic analyses of medical diagnostic thinking. Cogn Sci. 1992;16: 185-204.

4. Feltovich PJ, Barrows HS. Issues of generality in medical problem solving. In: Schmidt H, De Volder ML, editors. Tutorials in prob- lem-based learning: a new direction in teaching the health professions. Assen: Van Gorcum; 1984.

5. Charlin B, Boshuizen H, Custers E, Feltovitch P. Scripts and clinical reasoning. Med Educ. 2007;41:1178-84.

6. Custers EJFM. Thirty years of illness scripts: theoretical origins and practical applications. Med Teach. 2015;37:457-62.

7. Lubarsky S, Dory V, Duggan P, Gagnon R, Charlin B. Script concordance testing: from theory to practice: AMEE guide no. 75 . Med Teach. 2013;35:184-93.

8. Norman GR. Objective measurement of clinical performance. Med Educ. 1985;19:43-7.

9. Norcini JJ, Shea JA, Day SC. The use of the aggregate scoring for a recertification examination. Eval Health Prof. 1990;13:241-51.

10. Charlin B, Brailovsky CA, Leduc C, Blouin D. The diagnosis script questionnaire: a new tool to assess a specific dimension of clinical competence. Adv Health Sci Educ Theory Pract. 1998;3:51-8.

11. Lubarsky S, Charlin B, Cook DA, Chalk C, van der Vleuten C. Script concordance testing: a review of published validity evidence. Med Educ. 2011;45:329-38.

12. Cook DA, Beckman TJ. Current concepts in validity and reliability for psychometric instruments: theory and application. Am J Med. 2006;119:166.e7-166.e16.

13. Gagnon R, Charlin B, Roy L, et al. The cognitive validity of the script concordance test: a time processing study. Teach Learn Med. 2006;18:22-7.

14. Ahmadi SF, Khoshkish S, Soltani-Arabshahi K. Challenging script concordance test reference standard by evidence: do judgments by emergency medicine consultants agree with likelihood ratios? Int J Emerg Med. 2014;7:34.

15. Kreiter C. Commentary: the response process validity of a script concordance item. Adv Health Sci Educ Theory Pract. 2011;17:7-9.

16. Lineberry M, Kreiter CD, Bordage G. Threats to the validity in the use and interpretation of script concordance test scores. Med Educ. 2013;47:1175-83.

17. Wilson AB, Pike GR, Humbert A. Preliminary factor analyses raise concerns about script concordance test utility. Med Sci Educ. 2014; https://doi.org/10.1007/s40670-014-0013-6.

18. Bland A, Kreiter C, Gordon J. The psychometric properties of five scoring methods applied to the Script Concordance Test. Acad Med. 2005;80:395-9.

19. Gagnon R, Charlin B, Coletti M, Sauve E, van der Vleuten C. Assessment in the context of uncertainty: how many members are needed on the panel of reference of a script concordance test? Med Educ. 2005;39:284-91.

20. Lambert C, Gagnon R, Nguyen D, Charlin B. The script concordance test in radiation oncology: validation study of a new tool to assess clinical reasoning. Radiat Oncol. 2009;4:7.

21. Lubarsky S, Chalk C, Kazitani D, Gagnon R, Charlin B. The Script Concordance Test: a new tool assessing clinical judgement in neurology. Can J Neurol Sci. 2009;36:326-31.

22. Nouh T, Boutros M, Gagnon R, et al. The script concordance test as a measure of clinical reasoning: a national validation study. Am J Surg. 2012;203:530-4.

23. Wilson AB, Pike GR, Humbert A. Analyzing script concordance test: scoring methods and items by difficulty and type. Teach Learn Med. 2014;26:135-45.

24. Downing SM. Threats to the validity of locally developed multiplechoice tests in medical education: construct-irrelevant variance and construct underrepresentation. Adv Health Sci Educ Theory Pract. 2002; 7:235-41.

25. Williams RG, Klamen DA, McGaghie WC. Special article: cognitive, social and environmental sources of bias in clinical performance ratings. Teach Learn Med. 2003;15:270-92.

26. See KC, Tan KL, Lim TK. The script concordance test for clinical reasoning: re-examining its utility and potential weakness. Med Educ. 2014;48:1069-77. 
27. Fournier JP, Demeester A, Charlin B. Script concordance tests: guidelines for construction. BMC Med Inform Decis Mak. 2008;8: 18.

28. Friedman Ben-David M. Principles of assessment. In: Dent J, Harden RM, editors. A practical guide for medical teachers. 2nd ed. Edinburgh, Churchill, Livingstone: Elsevier; 2005.

29. Boulouffe C, Charlin B, Vanpee D. Evaluation of clinical reasoning in basic emergencies using a script concordance test. Am J Pharm Educ. 2010;74:1-6.

30. Ramaekers S, Kremer W, Pilot A, van Keulen H. Assessment of competence in clinical reasoning and decision-making under uncertainty: the script concordance test method. Assess Eval High Educ. 2010;35:661-73.

31. Dawson T, Comer L, Kossick MA, Neubrander J. Can script concordance testing be used in nursing education to accurately assess clinical reasoning skills? J Nurs Educ. 2014;53:281-6.

32. Van den Broek WES, van Asperen MV, Custers EJFM, Valk GD, ten Cate O. Effects of two different instructional formats on scores and reliability of a script concordance test. Perspect Med Educ. 2012;1:119-28.

Stuart Lubarsky MD, MHPE, is assistant professor of Neurology at McGill University and a core member of the McGill Centre for Medical Education. His current research interests include clinical reasoning and assessment of competence.
Valérie Dory MD, PhD, MMedEd, is assistant professor at the Faculty of Medicine and the Centre for Medical Education. She is now the lead for Assessment and Evaluation in Undergraduate Medical Education at McGill. Her current research interests include assessment, particularly of clinical reasoning, as well as epistemological beliefs and their development in medical school.

Sarkis Meterissian MD, MA (Ed), is assistant professor in Radio-Oncology and member of the Centre de pédagogie appliquée aux sciences de la santé (CPASS) at the Centre Hospitalier de l'Université de Montréal. Her current research interests include script concordance testing and competency based medicine.

Carole Lambert MD, MA(Ed), is assistant professor in Radio-Oncology and member of the Centre de pédagogie appliquée aux sciences de la santé (CPASS) at the Centre Hospitalier de l'Université de Montréal. Her current research interests include script concordance testing and competency based medicine.

Robert Gagnon MPsy, is the director of the Assessment Office and member of the Centre de pédagogie appliquée aux sciences de la santé (CPASS) in the Faculty of Medicine at the University of Montréal. His research interests include assessment of clinical reasoning and test construction. 